\title{
A COVID-19 E OS DESAFIOS PARA O SISTEMA E OS PROFISSIONAIS DE SAÚDE
}

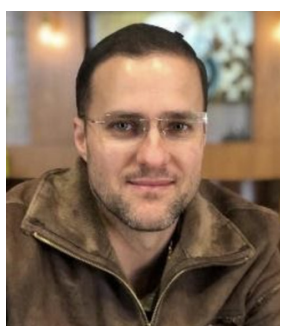

\section{Luciano Garcia Lourenção}

Professor Titular-Livre - Universidade Federal do Rio Grande

Editor Associado - Revista Enfermagem em Foco

ORCID - https://orcid.org/0000-0002-1240-4702

Nas últimas décadas acompanhamos o aparecimento de novos e importantes problemas de saúde, oriundos das mudanças socioeconômicas, tecnológicas, ambientais e demográficas, que causaram alterações no perfil das populações. Nesse contexto, enquanto a transição demográfica nos impõe a necessidade de estruturar o sistema de saúde para o enfrentamento das doenças crônicas não transmissiveis, inerentes ao processo de envelhecimento, o contexto epidemiológico faz com que doenças transmissiveis persistam entre as dez principais causas de morte no mundol.

As alterações no perfil de morbidade e mortalidade causadas pela associação entre a transição demográfica e a epidemiológica exigem, além de uma competente e arrojada Rede de Atenção à Saúde (RAS), o emprego de tecnologias que atendam as demandas populacionais, nos diferentes niveis de atendimento. No entanto, incoerências entre as necessidades de saúde de uma população e a forma como as respostas político-organizacionais são estruturadas podem ocasionar grandes crises nos sistemas de atenção à saúde2, aumentando as demandas por serviços especializados de elevado custo e de maior densidade tecnológica.

O subfinanciamento e a ineficiência na gestão são desafios que assombram a capacidade de respostas às demandas do Sistema Único de Saúde (SUS), responsável pela assistência direta à saúde de mais de $80 \%$ dos brasileiros. Além da má gestão dos recursos, a situação política e econômica que se instalou no Brasil, nos últimos anos, levou a maiores cortes de financiamento, sobretudo no Setor Saúde, e ao aumento do desemprego e da informalidade, que favorece a expansão da linha da pobreza, levando ao aumento de agravos e doenças, sobrecarregando os serviços de saúde e agravando ainda mais a problemática do acesso e da resolubilidade do sistema público de saúde3-4.

Não obstante, nos últimos anos nos deparamos com o recrudescimento de doenças transmissiveis emergentes e reemergentes, como a Dengue, Influenza $A(\mathrm{H} I N 1)$, Zika,
Febre Chikungunya e, mais recentemente, a COVID-19, com potencial para colapsar os sistemas de saúde, em caso de epidemias, pois encontram grande número de pessoas susceptiveis, a ausência de medidas profiláticas efetivas, vacinas eficazes e/ou tratamentos específicos.

A preocupação mais recente é a COVID-19, uma síndrome respiratória aguda grave causada pelo novo coronavírus SARS-COV-2, que teve origem na cidade chinesa de Wuhan, no final de 2019, e rapidamente se disseminou por países de todo o mundo, sendo declarada pela Organização Mundial de Saúde (OMS) uma Emergência de Saúde Pública de Importância Internacional (ESPII), em 30 de janeiro de 2020. No Brasil, foi declarada Emergência em Saúde Pública de Importância Nacional (ESPIN) em 3 de fevereiro de 2020 e, no dia 26, o país confirmou o primeiro caso da doença, o que agravou a preocupação das autoridades sobre seus impactos na saúde dos brasileiros e suas consequências políticas, sociais, econômicas e sanitárias1,5-6.

Entre as muitas dúvidas sobre a doença, há a preocupação com a capacidade de atendimento dos serviços de saúde, dada a estimativa de que $20 \%$ das pessoas acometidas pela doença necessitem de assistência médico-hospitalar e cerca de $5 \%$ precisem de cuidados intensivos de alta complexidade7.

$\mathrm{Na}$ linha de frente de toda essa possivel demanda de atendimento aos pacientes contaminados pelo novo coronavírus estão os profissionais da saúde, em especial, os trabalhadores de Enfermagem, que somam mais de 2,3 milhões, em todo o país. A depender da dimensão que esta pandemia tomará no Brasil, milhares de profissionais poderão se contaminar ou até mesmo morrer, em decorrência do trabalho.

$\mathrm{Na}$ iminência de um colapso do sistema de saúde, além do risco de morte, os profissionais de enfermagem sofrerão estresse intenso e o consequente risco do adoecimento mental, situação que exige atenção especial dos gestores e órgão de classe. Nesse contexto o COFEN, juntamente com os Conselhos Regionais de Enfermagem, está atento, monitorando as condições de trabalho dos profissionais e exigindo condições adequadas e seguras, com fornecimento de Equipamentos de Proteção de Individual, dimensionamento adequado dos trabalhadores e afastamento daqueles que se encontram nos grupos de risco.

No mundo inteiro, a COVID-19 tem desafiado governos, pesquisadores, profissionais da saúde e gestores a encontrar 
estratégias de preservação da vida. Sem perspectiva da aprovação de uma vacina para conter a disseminação do vírus ou de um medicamento eficaz e seguro para tratar a doença, a melhor estratégia é retardar sua transmissão, evitando a sobrecarga dos serviços de saúde.

Epidemiologicamente, podemos dizer que a taxa de disseminação da infecção por SARS-COV-2 dependerá do tempo de duração da infecção - estimado em 14 dias, em média -, do número de pessoas para as quais um paciente pode transmitir a doença, das chances de cada oportunidade se concretizar em transmissão e da susceptibilidade da população à doença8.

Considerando que nossa capacidade de mudar o tempo de duração da infecção é quase nula e que o número de pessoas susceptíveis, enquanto não houver vacina, depende do contato com a doença, é imprescindivel que reforcemos as medidas preventivas, como lavagem das mãos, uso de máscaras e do álcool em gel, e evitemos o máximo possível os contatos sociais não essenciais. Dessa forma, contribuiremos para prolongar o tempo de propagação da doença, evitando sobrecarga dos serviços de saúde e preservando vidas.

Enquanto isso, compete aos pesquisadores buscar uma vacina e medicamentos eficazes contra a doença, que possam ajudar a preservar vidas e reduzir impactos sociais, econômicos e sanitários desta grave epidemia.

$\mathrm{E}$ aos gestores, cabe a responsabilidade de estruturar os serviços de saúde, para receber os doentes, e garantir condições de trabalho para que os profissionais da saúde, entre eles, os profissionais de Enfermagem, prestem cuidados de qualidade e com segurança, contribuindo para salvar vidas!

Descritores: Infecções por coronavírus; Doenças transmissíveis; Enfermagem; Sistemas de Saude.

COVID-19 AND THE CHALLENGES FOR THE SYSTEM AND HEALTH PROFESSIONALS

Descriptors: Coronavirus Infections; Communicable Diseases; Nursing; Health Sistems. COVID-19 Y LOS DESAFÍOS PARA EL SISTEMA Y LOS PROFESIONALES DE LA SALUD

Descriptores: Infecciones por coronavirus; Enfermedades transmisibles; Enfermería; Sistemas de Salud.

\section{REFERÊNCIAS}

1. World Health Organization. Ending the epidemics of high impact communicable diseases. 2018 [cited 2020 Feb 28]. Available from: www.who.int/news-room/commentaries/detail/ending-the-epidemics-of-high-impact-communicable-diseases.

2. Duarte E, Eble LJ, Garcia LP. 30 anos do Sistema Único de Saúde. Epidemiol. Serv. Saúde. [Internet]. 2018 [cited 2020 Feb 28]; 27(1):e00100018. Available from: http://dx.doi.org/10.5123/ s1679-49742018000100018.

3. Almeida ER, Sousa ANA, Brandão CC, Carvalho FFB, Tavares G, Silva KC. Política Nacional de Atenção Básica no Brasil: uma análise do processo de revisão (2015-2017). Rev Panam Salud Publica. [Internet]. 2018 [cited 2020 Feb 28];42:el80. Available from: https://doi.org/10.26633/RPSP.2018.180

4. Sales OP, Vieira AFB, Martins AM, Garcia LG, Ferreira RKA. O Sistema Único de Saúde: desafios, avanços e debates em 30 anos de história. Humanidades \& Inovação. [Internet]. 2019 [cited 2020 Feb 28];6(17):54-65. Available from: https://revista.unitins.br/index.php/humanidadeseinovacao/article/view/1045.

5. World Health Organization. Statement on the second meeting of the International Health Regulations (2005) Emergency
Committee regarding the outbreak of novel coronavirus (2019nCoV). Geneva: World Health Organization; 2020 [cited 2020 Feb 28]. Available from: https://www.who.int/news-room/detail/30-01-2020-statement-on-the-second-meetingof-the-international-health-regulations-(2005)-emergency-committee-regarding-the-outbreak-of-novel-coronavirus-(2019-ncov).

6. Ministério da Saúde (BR). Portaria MS/GM n. 188, de 3 de fevereriro de 2020. Declara Emergência em Saúde Pública de importância Nacional (ESPIN) em decorrência da Infecção Humana pelo novo Coronavirus (2019-nCoV). Diário Oficial da União, Brasilia (DF), 2020 fev 4 [cited 2020 Feb 28]; Seção 1:1. Available from: http://www.in.gov.br/web/dou/-/portaria-n-188-de-3-de-fevereiro-de-2020-241408388.

7. Zhou F, Yu T, Du R, Fan G, Liu Y, Liu Z et al. Clinical course and risk factors for mortality of adult inpatients with COVID-19 in Wuhan, China: a retrospective cohort study. Lancet. [Internet]. 2020 [cited 2020 Mar 13];395(10229):1054-1062. Available from: https://doi.org/10.1016/S0140-6736(20)30566-3.

8. Kucharski A. The Rules of Contagion: why things spread and why they stop. Basic Books: New York; 2020. 